\section{nephron \\ Clinical}

Practice
Nephron 2017; 137:15-22

DOI: $10.1159 / 000473704$
Received: January 11, 2017

Accepted after revision: March 27, 2017

Published online: April 27, 2017

\title{
Early Mortality Associated with Inpatient versus Outpatient Hemodialysis Initiation in a Large Cohort of US Veterans with Incident End-Stage Renal Disease
}

\author{
Faisal M. Arif ${ }^{a}$ Keiichi Sumida ${ }^{a}$ e, $f$ Miklos Z. Molnar ${ }^{a}$ g Praveen K. Potukuchi $^{a}$ \\ Jun Ling Lu ${ }^{a}$ Fatima Hassan $^{a}$ Fridtjof Thomas ${ }^{b}$ Omer A. Siddiqui ${ }^{a}$ \\ Geeta G. Gyamlanic Kamyar Kalantar-Zadeh ${ }^{d}$ Csaba P. Kovesdya, c \\ aDivision of Nephrology, Department of Medicine, and ${ }^{b}$ Division of Biostatistics, Department of Preventive Medicine, \\ University of Tennessee Health Science Center, and 'Nephrology Section, Memphis VA Medical Center, Memphis, TN, \\ and ${ }^{\mathrm{d}}$ Harold Simmons Center for Chronic Disease Research and Epidemiology, Division of Nephrology and \\ Hypertension, University of California-Irvine, Orange, CA, USA; ${ }^{~}$ Nephrology Center, Toranomon Hospital Kajigaya, \\ Kanagawa, and ${ }^{\mathrm{f} D e p a r t m e n t}$ of Nephrology, Faculty of Medicine, University of Tsukuba, Ibaraki, Japan; ${ }^{9}$ Department \\ of Transplantation and Surgery, Semmelweis University, Budapest, Hungary
}

\section{Keywords}

Hospitalization · Chronic kidney disease $\cdot$ End-stage renal disease $\cdot$ Mortality

\begin{abstract}
Background: Mortality in the immediate post-hemodialysis transition period is extremely high. Many end-stage renal disease (ESRD) patients in the US start dialysis in an inpatient setting, but the characteristics of patients starting dialysis as inpatients, and the association of inpatient hemodialysis transition with mortality remain unclear. Methods: We examined 48,261 US veterans who transitioned to hemodialysis between October 2007 and September 2011. Associations of inpatient hemodialysis starting with all-cause mortality were examined in Cox proportional hazard models, with adjustments for demographics, comorbidities, vascular access type, pre-dialysis nephrology care and medication use, and last pre-ESRD estimated glomerular filtration rate
\end{abstract}

\section{KARGER}

() 2017 S. Karger AG, Basel

E-Mail karger@karger.com

www.karger.com/nef and hemoglobin. Results: A total of 22,338 (46.3\%) patients received the first hemodialysis treatment in an inpatient setting. Inpatient hemodialysis transition was associated with older age, presence of a tunneled catheter, higher comorbidity burden, and lack of pre-dialysis nephrology care. A total of 8,674 patients died (mortality rate 405/1,000 patientyears, $95 \% \mathrm{Cl} 397-413$ ) during the first 6 months after transition to hemodialysis. The starting of inpatient vs. outpatient hemodialysis was associated with significantly higher crude all-cause mortality, but this association was attenuated after multivariable adjustments. Conclusions: Transition to hemodialysis in an inpatient setting is more common in older and sicker individuals, and in patients without pre-dialysis nephrology care and those who used a catheter for vascular access. Future studies are needed to determine if a higher proportion of patients could start hemodialysis treatment in outpatient clinics, through interventions targeting modifiable risk factors such as timely vascular access placement or earlier nephrology referrals.

(c) 2017 S. Karger AG, Basel
Dr. Csaba P. Kovesdy

Nephrology Section, Memphis VA Medical Center 1030 Jefferson Ave

Memphis, TN 38104 (USA)

E-Mail ckovesdy@uthsc.edu 


\section{Introduction}

Each year, more than 100,000 patients transition to chronic maintenance hemodialysis in the US [1]. Starting renal replacement therapy is an important and often lifechanging event for patients, which also greatly impacts their survival [2]. The decision about the best way to start renal replacement therapy is a complex one involving considerations of patient characteristics and preferences, physician practice characteristics, and a dynamic interaction between physicians and patients [3]. An optimal transition to renal replacement therapy usually requires that patients are referred to a nephrologist, a modality choice is made after proper patient education, a dialysis access is placed in a timely manner, and close follow-up is provided to manage the consequences of progressively deteriorating kidney function, culminating in a decision to initiate the preferred renal replacement therapy. Due to the natural uncertainty of when any individual with advanced chronic kidney disease (CKD) might reach the point where dialysis needs to be initiated, there is still considerable uncertainty about the best timing and the optimal circumstances of initiating hemodialysis.

During the last few decades, there has been a changing trend in the timing of the initiation of hemodialysis in advanced CKD. There has been considerable debate over whether or not hemodialysis should be initiated early (based solely on the level of estimated glomerular filtration rate [eGFR]) $[4,5]$ or late (based on the level of kidney function or other indications such as uremic symptoms or electrolyte abnormalities) [6, 7]. A recent randomized clinical trial indicated that early hemodialysis start portends no survival advantage, and that waiting for an actual medical indication did not carry a significantly increased risk for late starters [8]. Notwithstanding the safety of late hemodialysis start in a clinical trial setting, waiting for medical indications before transitioning to hemodialysis carries the risk of acute medical complications, which may require urgent interventions, often in a hospital setting, and may make planned hemodialysis initiation more difficult. Unplanned, urgent hemodialysis initiation is associated with worse outcomes compared to planned, elective hemodialysis initiation [9], but the reasons behind urgent and emergent hemodialysis initiation remain less well studied on a population level, in part because large administrative databases typically do not provide sufficiently detailed information about medical indications for hemodialysis initiation.

One aspect of urgent or emergent hemodialysis transition is that it occurs in an inpatient hospital setting, but hospitalizations for hemodialysis transition may also be required in cases when a hemodialysis vascular access is not available, or occasionally even for administrative reasons. Notwithstanding the uncertainty of why an individual patient may transition to hemodialysis in an inpatient hospital setting, hospitalizations carry the risk of iatrogenic complications (e.g., nosocomial infections), and may contribute to the extremely high short-term mortality experienced by hemodialysis patients during the first 3-6 months after their transition [1]. Furthermore, inpatient transition to hemodialysis may also impose significantly increased costs [10], thus providing an added impetus to favor elective, outpatient hemodialysis transition in most patients.

Despite these considerations, there is a paucity of data about the reasons behind inpatient hemodialysis transition in the US, and whether or not the setting of hemodialysis transition (inpatient vs. outpatient) has an independent effect on subsequent patient outcomes. In order to inform about these, we examined the association of different patient characteristics with the setting of hemodialysis transition, and the association between the setting of transition and subsequent short-term mortality in a large cohort of US veterans.

\section{Materials and Methods}

\section{Cohort Definition}

We examined data obtained from the Transition of Care in CKD study, a retrospective cohort study of 52,172 US veterans with incident end-stage renal disease (ESRD) who transitioned to renal replacement therapy from October 1, 2007, through September 30, 2011, as identified from the United States Renal Data System (USRDS) Patient and Medical Evidence Form 2728 [11-13]. We excluded patients who initiated renal replacement therapy with peritoneal dialysis $(n=2,401)$, pre-emptive transplants $(n=$ $585)$, or an unknown modality $(n=737)$, or who died the same day renal replacement therapy was started $(n=188)$, resulting in a final cohort of 48,261 patients (Fig. 1).

\section{Data Collection}

We identified inpatient hemodialysis transition by matching the hemodialysis first service date with individual hospitalization dates obtained from VA Inpatient Medical SAS Datasets and from Centers for Medicare and Medicaid Services (CMS) data files. The same data sources were also used to extract information about the number and duration of all inpatient hospitalizations during the prelude period (i.e., prior to hemodialysis start). Data from the USRDS Patient and Medical Evidence form were used to determine baseline demographic characteristics, vascular access type at the time of dialysis initiation and information about the last eGFR and hemoglobin concentrations prior to hemodialysis start. Data on prevalent comorbidities was obtained from the VA Inpatient and Outpatient Medical SAS Datasets and from CMS data files,
16

Nephron 2017;137:15-22 DOI: $10.1159 / 000473704$
Arif et al. 


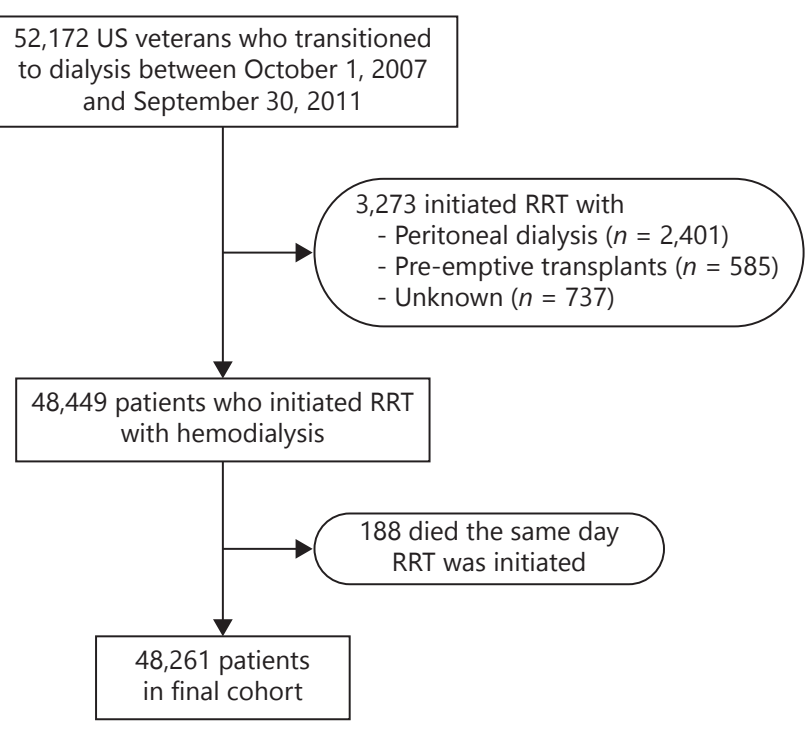

Fig. 1. Algorithm of cohort definition.

using ICD-9-CM diagnostic and procedure codes and CPT codes recorded during the hemodialysis prelude period, as previously described [14]. Cardiovascular disease was defined as the presence of diagnostic codes for coronary artery disease, angina, myocardial infarction, or cerebrovascular disease. The Charlson Comorbidity Index (CCI) score was calculated using the Deyo modification for administrative data sets, without including kidney disease [15]. Medication use during the prelude period was extracted from VA Pharmacy dispensation records and from CMS (Medicare Part D) files [16].

\section{Statistical Analysis}

Data are presented as number (percent), means \pm SD or median (interquartile range [IQR]), as appropriate. Differences between patients categorized according to inpatient vs. outpatient hemodialysis transition were assessed by $t$ test, the Mann-Whitney $U$ test or chi-square test, as appropriate. The association of various patient characteristics with inpatient vs. outpatient hemodialysis transition was examined in multivariable logistic regression models.

The primary outcome was all-cause mortality during the first 6 months after hemodialysis initiation. Information about allcause mortality was obtained from the VA Vital Status Files [17]. We examined the association of inpatient vs. outpatient hemodialysis transition with all-cause mortality during the first 6 months following transition using the Kaplan-Meier method and the log-rank test, and we calculated hazards ratios (HRs) and 95\% CI in unadjusted and multivariable adjusted Cox models. Multivariable models were adjusted based on theoretical considerations for age, gender, race, individual comorbid conditions (history of diabetes mellitus, myocardial infarction, congestive heart failure, peripheral vascular disease, cerebrovascular disease, dementia, chronic lung disease, liver disease, and malignancies), the CCI as an omnibus measure of illness, receipt of

Inpatient vs. Outpatient Hemodialysis Transition nephrology subspecialty care during the prelude period, use of medications during the prelude period (angiotensin converting enzyme inhibitors of angiotensin receptor blockers, statins, erythropoietin), and active (calcitriol, paricalcitol, or doxercalciferol) and nutritional (ergocalciferol or cholecalciferol) vitamin D, the last eGFR and hemoglobin level prior to hemodialysis start, and the number and average length of hospitalizations during the prelude period.

Of the variables included in multivariable models, data points were missing for race $(0.1 \%)$, access type $(1.4 \%)$, comorbid conditions $(9.2 \%)$, pre-dialysis nephrology care $(13.3 \%)$, eGFR $(3.7 \%)$, and blood hemoglobin concentration (11.2\%). A total of 33,316 patients $(69 \%)$ had complete data for multivariable analysis. We did not impute missing data in our primary multivariable models, and used multiple imputations to replace missing variables in sensitivity analyses. In additional sensitivity analyses, we also examined mortality in Cox models over a 1-year post-transition time period and after the initial 6-month and 1-year in patients who survived these respective time periods. Analyses were conducted using STATA MP Version 14 (STATA Corporation, College Station, TX, USA). The study was approved by the Institutional Review Boards of the Memphis and Long Beach VA Medical Centers, with exemption from informed consent.

\section{Results}

Of the 48,261 patients starting hemodialysis, 22,338 (46\%) transitioned in an inpatient setting. The median (IQR) length of hospitalization for patients initiating hemodialysis as inpatients was 10 (7-17) days. In these patients, hemodialysis was started a median (IQR) of 3 (27) days after hospital admission, and patients were discharged from the hospital a median (IQR) of 6 (3-10) days after the first hemodialysis treatment; 2,432 patients (11\%) were discharged the day after the first hemodialysis treatment. Patients' baseline characteristics at the time of dialysis initiation overall and by hemodialysis start setting (inpatient vs. outpatient) are presented in Table 1. Overall, patients were $70.6 \pm 11.9$ years old, $95 \%$ were men, 25\% were African-American, and 58\% had diabetes. Compared to patients who started hemodialysis as outpatients, those transitioning in an inpatient setting were older, less likely to be female and black, had a higher prevalence of comorbid conditions, were less likely to have received pre-transition nephrology care, were more likely to use a tunneled dialysis catheter (vs. an arteriovenous fistula, AVF), and had a higher number and longer average duration of hospitalizations in the prelude period.

In a multivariable logistic regression analysis older age, tunneled hemodialysis catheter use, congestive heart failure, a higher number and longer pre-dialysis hospi-

Nephron 2017;137:15-22 
Table 1. Baseline characteristics

\begin{tabular}{|c|c|c|c|c|}
\hline & $\begin{array}{l}\text { Overall } \\
(n=48,213)\end{array}$ & $\begin{array}{l}\text { Inpatients } \\
(n=22,338)\end{array}$ & $\begin{array}{l}\text { Outpatients } \\
(n=25,875)\end{array}$ & $p$ value \\
\hline Age, years & $70.6 \pm 11.9$ & $73.1 \pm 10.7$ & $68.5 \pm 12.6$ & $<0.001$ \\
\hline Gender, male & $45,630(94)$ & $21,309(95)$ & $24,321(94)$ & $<0.001$ \\
\hline Race: black & $11,877(25)$ & $4,915(22)$ & $6,962(27)$ & $<0.001$ \\
\hline Ethnicity: hispanic & $2,877(6)$ & $1,247(6)$ & $1,630(6)$ & 0.001 \\
\hline Myocardial infarction & $12,605(29)$ & $7,656(35)$ & $4,949(23)$ & $<0.001$ \\
\hline Congestive heart failure & $25,061(58)$ & $14,900(69)$ & $10,161(48)$ & $<0.001$ \\
\hline Peripheral vascular disease & $17,331(40)$ & $9,809(45)$ & $7,522(36)$ & $<0.001$ \\
\hline Cerebrovascular disease & $13,606(32)$ & $7,706(35)$ & $5,900(28)$ & $<0.001$ \\
\hline Dementia & $1,297(3)$ & $794(4)$ & $503(2)$ & $<0.001$ \\
\hline Lung diseases & $19,370(45)$ & $11,199(52)$ & $8,171(39)$ & $<0.001$ \\
\hline Diabetes mellitus & $28,110(58)$ & $14,756(66)$ & $13,354(52)$ & $<0.001$ \\
\hline Liver disease & $5,086(11)$ & $2,872(13)$ & $2,214(9)$ & $<0.001$ \\
\hline Cancer & $11,041(23)$ & $6,115(27)$ & $4,926(19)$ & $<0.001$ \\
\hline Received pre-ESRD nephrology care & $29,204(70)$ & $12,074(63)$ & $17,130(75)$ & $<0.001$ \\
\hline Last eGFR before ESRD, $\mathrm{mL} / \mathrm{min} / 1.73 \mathrm{~m}^{2}$ & $12 \pm 5$ & $12 \pm 5$ & $12 \pm 5$ & $<0.001$ \\
\hline Hemoglobin, g/dL & $10.0 \pm 1.6$ & $10.0 \pm 1.5$ & $10.1 \pm 1.6$ & $<0.001$ \\
\hline ACEI & $27,436(57)$ & $13,833(62)$ & $13,603(52)$ & $<0.001$ \\
\hline EPO & $9,177(19)$ & $4,427(20)$ & $4,750(18)$ & $<0.001$ \\
\hline Statin & $26,586(55)$ & $13,459(60)$ & $13,127(51)$ & $<0.001$ \\
\hline Active vitamin D & $11,148(23)$ & $4,864(22)$ & $6,284(24)$ & $<0.001$ \\
\hline Regular vitamin D & $5,110(10.6)$ & $2,347(11)$ & $2,763(11)$ & 0.6 \\
\hline CCI & $4.8 \pm 3.0$ & $5.3 \pm 2.9$ & $4.2 \pm 2.9$ & $<0.001$ \\
\hline Vascular access type & & & & $<0.001$ \\
\hline AVF & $8,993(19)$ & $2,103(9)$ & $6,890(27)$ & \\
\hline AVG & $1,245(3)$ & $353(2)$ & $892(4)$ & \\
\hline Catheter & $37,021(78)$ & $19,638(88)$ & $17,383(69)$ & \\
\hline Length of hospitalizations during prelude, days & $6(9-4)$ & $6.8(4.8-10)$ & $4.7(3-7)$ & $<0.001$ \\
\hline Number of hospitalizations during prelude & $3(1-6)$ & $4(2-8)$ & $1(0-4)$ & $<0.001$ \\
\hline
\end{tabular}

Values are, $n(\%)$ or mean \pm SD.

ACEi, angiotensin converting enzyme inhibitors and angiotensin receptor blockers; AVF, arteriovenous fistula; AVG, arteriovenous graft; EPO, erythropoetin; ESRD, end-stage renal disease; CCI, Charlson comorbidity index.

talizations, and not having received nephrology care in the pre-dialysis period were associated with higher odds of inpatient (vs. outpatient) dialysis initiation. Conversely, higher eGFR and blood hemoglobin concentration, and use of erythropoietin and active vitamin D during the prelude period were associated with lower odds of inpatient (vs. outpatient) hemodialysis initiation (Table 2).

A total of 8,674 patients died (mortality rate 405/1,000 patient-years, 95\% CI 397-413) during the first 6 months after transition to hemodialysis, and 32,323 patients died overall during a median follow-up time of 2.1 years (mortality rate: 290/1,000 patient-years, 95\% CI 287-293). Figure 2 shows cumulative mortality curves for all-cause mortality during the first 6 months in patients divided by inpatient vs. outpatient hemodialysis initiation status, in- dicating higher unadjusted risk of all-cause mortality in patients who started hemodialysis as inpatients. Table 3 shows unadjusted and multivariable adjusted HRs and $95 \%$ CI of all-cause mortality associated with inpatient vs. outpatient hemodialysis start in the first 6 months and 1 year after transition for hemodialysis, and following the initial 6-month and 1-year periods in individuals who survived these respective periods. Inpatient hemodialysis start was associated with higher unadjusted 6-month mortality risk (crude HR and 95\% CI 1.90 (1.82-1.99), $p<$ 0.001 ), which was substantially attenuated after multivariable adjustment (multivariable adjusted $\mathrm{HR}$ and 95\% CI 1.07 (1.01-1.13), $p=0.02$ ). Similar tendencies were observed for 1-year mortality, while in patients who survived the initial 6 -month and 1-year time periods, the multivariable adjusted mortality risk was not different in 
Table 2. Variables associated with inpatient hemodialysis initiation

\begin{tabular}{|c|c|c|c|}
\hline & OR & $95 \% \mathrm{CI}$ & $p$ value \\
\hline Age (10 years higher) & 1.14 & $1.11-1.17$ & $<0.001$ \\
\hline Gender (female vs. male) & 0.98 & $0.88-1.10$ & 0.05 \\
\hline Race (black vs. white) & 1.02 & $0.96-1.08$ & 0.5 \\
\hline \multicolumn{4}{|l|}{ Vascular access } \\
\hline AVG (vs. AVF) & 1.20 & $1.03-1.40$ & 0.02 \\
\hline Catheter (vs. AVF) & 3.22 & $3.02-3.44$ & $<0.001$ \\
\hline MI (presence vs. absence) & 1.20 & $1.12-1.26$ & $<0.001$ \\
\hline CHF (presence vs. absence) & 1.76 & $1.67-1.86$ & $<0.001$ \\
\hline PVD (presence vs. absence) & 1.07 & $1.02-1.13$ & 0.01 \\
\hline CVD (presence vs. absence) & 1.03 & $0.98-1.09$ & 0.3 \\
\hline Dementia (presence vs. absence) & 1.12 & $0.97-1.29$ & 0.1 \\
\hline Lung diseases (presence vs. absence) & 1.14 & $1.08-1.20$ & $<0.001$ \\
\hline Diabetes (presence vs. absence) & 1.03 & $0.97-1.09$ & 0.4 \\
\hline Cancer (presence vs. absence) & 1.01 & $0.94-1.08$ & 0.8 \\
\hline Liver disease (presence vs. absence) & 1.15 & $1.07-1.25$ & $<0.001$ \\
\hline CCI (1 point higher) & 1.05 & $1.04-1.07$ & $<0.001$ \\
\hline No Nephrology Care & 1.50 & $1.42-1.59$ & $<0.001$ \\
\hline eGFR, $1 \mathrm{~mL} / \mathrm{min} / 1.73 \mathrm{~m}^{2}$ higher & 1.00 & $0.99-1.00$ & 0.2 \\
\hline Hemoglobin, $1 \mathrm{~g} / \mathrm{dL}$ higher & 0.89 & $0.87-0.90$ & $<0.001$ \\
\hline ACEI use & 0.96 & $0.91-1.02$ & 0.2 \\
\hline EPO use & 1.06 & $1.00-1.13$ & 0.06 \\
\hline Statin use & 0.96 & $0.91-1.02$ & 0.2 \\
\hline \multicolumn{4}{|l|}{ Vitamin D use } \\
\hline Active & 0.90 & $0.85-0.96$ & 0.001 \\
\hline Regular & 0.97 & $0.89-1.04$ & 0.4 \\
\hline \multicolumn{4}{|l|}{ Length of hospitalizations during } \\
\hline prelude (1 log-unit higher) & 3.71 & $3.54-3.89$ & $<0.001$ \\
\hline \multicolumn{4}{|l|}{ Number of hospitalizations during } \\
\hline prelude (1 log-unit higher) & 1.72 & $1.64-1.80$ & $<0.001$ \\
\hline
\end{tabular}

$\mathrm{ACEi}$, angiotensin converting enzyme inhibitors and angiotensin receptor blockers; AVF, arteriovenous fistula; AVG, arteriovenous graft; EPO, erythropoetin; ESRD, end stage renal disease; MI, myocardial infarction; $\mathrm{CHF}$, congestive heart failure; PVD, peripheral vascular disease; CVD, cerebrovascular disease; CCI, Charlson comorbidity index.

Fig. 2. Six-month all-cause mortality curves in patients transitioning to hemodialysis in an inpatient vs. an outpatient setting.

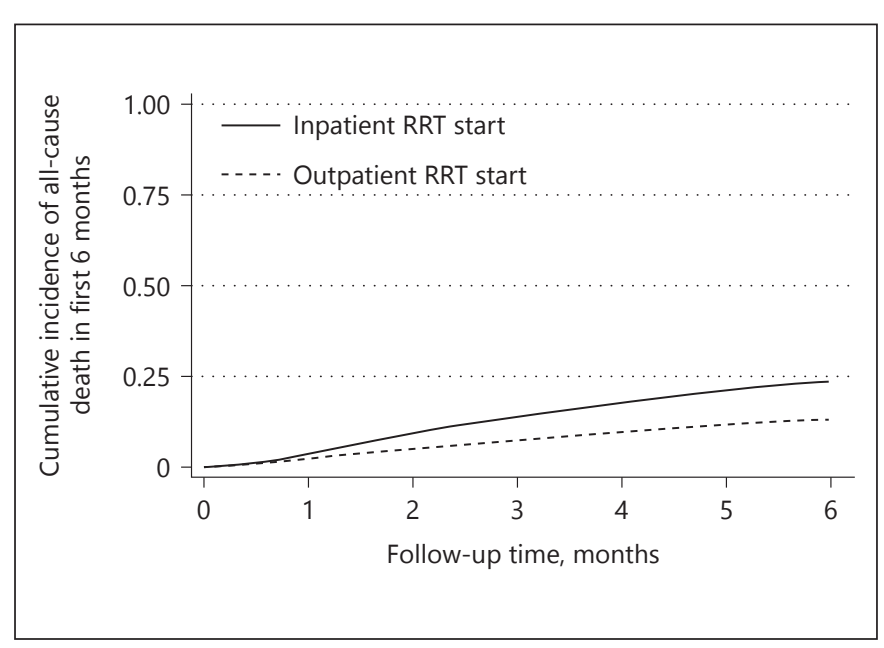

Nephron 2017;137:15-22 DOI: $10.1159 / 000473704$ 
Table 3. HRs and 95\% CIs of all-cause mortality following transition to hemodialysis during different follow-up periods

\begin{tabular}{cllll}
\hline & $\begin{array}{l}\text { Unadjusted HR } \\
(95 \% \mathrm{CI})\end{array}$ & $p$ value & $\begin{array}{l}\text { Adjusted HR } \\
(95 \% \mathrm{CI})\end{array}$ & $p$ value \\
\hline 0-6 months post transition & $1.90(1.82-1.96)$ & $<0.001$ & $1.07(1.01-1.13)$ & 0.02 \\
$>$ 6 months post transition & $1.43(1.40-1.47)$ & $<0.001$ & $1.01(0.97-1.04)$ & 0.62 \\
$0-12$ months post transition & $1.81(1.74-1.87)$ & $<0.001$ & $1.05(1.01-1.10)$ & 0.02 \\
$>12$ months post transition & $1.38(1.34-1.42)$ & $<0.001$ & $1.00(0.96-1.04)$ & 0.9 \\
\hline
\end{tabular}

patients who transitioned to hemodialysis as inpatients vs. outpatients (Table 3). Results remained unchanged in models using multiply imputed missing covariates (results not shown).

\section{Discussion}

In this large national retrospective cohort study of US veterans transitioning to hemodialysis in the hospital setting vs. in an outpatient setting, we found that patients who transitioned as inpatients were older, had a higher prevalence of congestive heart failure and dementia, and experienced higher numbers and longer hospitalizations during the hemodialysis prelude period. These associations suggest that inpatient transition to hemodialysis was in part driven by medical indications (e.g., patients with congestive heart failure developing fluid overload, or older patients experiencing more severe consequences of uremia requiring hemodialysis initiation in a higher acuity setting), and that a history of frequent and/or long hospitalizations is associated with a higher likelihood of needing to be in the hospital for the transition to hemodialysis. We also described an association of inpatient hemodialysis transition with less nephrology subspecialty care received prior to transition, and with the use of a catheter as vascular access. The latter associations underscore the importance of proper nephrology subspecialty care during the prelude (pre-transition period). Such care could result in a higher proportion of patients undergoing placement of an AVF, and could also result in various other benefits (e.g., better metabolic and volume control, or better patient education). Our description of associations between pre-transition hemoglobin and eGFR levels and of the use of certain medications and lower risk of inpatient hemodialysis transition might reflect effects of such better care received during hemodialysis prelude.
The proportion of patients transitioning to hemodialysis in an inpatient vs. an outpatient setting in our study (46.3\%) was lower than the $74.7 \%$ reported in a study of 1,691 US veterans whose medical charts were reviewed to identify the mechanisms of the decision-making underlying dialysis initiation [3]. By reviewing individual cases this study emphasized that decisions related to the timing of dialysis initiation is complex, and includes both patient-related and practitioner-related factors. Our observations showing associations of patient demographics, comorbidities, and pre-transition nephrology care with the setting of hemodialysis transition, support these conclusions. Our study also confirms and extends to a wider population the findings of a smaller prospective cohort of 652 incident hemodialysis patients, which reported that $63 \%$ of patients started hemodialysis in an inpatient setting, with the majority of hospitalizations being driven by medical indications such as uremia and volume overload [18].

The variables associated with inpatient hemodialysis transition suggest that this may potentially be avoidable in some patients by assuring proper pre-dialysis care, for example, by referring patients to a nephrologist and by timely creation of an AVF. Our data indicates that patients spend a relatively long time in the hospital during the hemodialysis transition period (median 10 days), presumably due to delays in placing a vascular access, or the need for treatment for conditions such as congestive heart failure. Better implementation of pre-transition nephrology care could conceivably reduce both the proportion of patients needing inpatient transition, and also the duration of the hospitalization in those admitted for hemodialysis transition, thus resulting in lower costs and potentially fewer hospitalization-related medical complications.

It is less clear if lowering the proportion of patients needing inpatient hemodialysis transition could result in better post-transition outcomes, such as lower mortality 
rates. In our study the unadjusted risk of early mortality associated with inpatient vs. outpatient hemodialysis transition was significantly higher, but the difference was almost completely abrogated by adjustment for various confounding characteristics. This suggests that the observed association between inpatient transition and mortality was in large part due to underlying patient characteristics and/or lack of proper pre-transition care, and not caused by the hospitalization per se. Receiving more intensive pre-dialysis nephrology care has been associated with more favorable outcomes [19], as has initiation of hemodialysis using an AVF (compared to an arteriovenous graft, and especially to a central venous catheter) as initial vascular access [20-22]. In addition to these welldescribed benefits of proper pre-transition nephrology care, our findings suggest that they may extend to lowering the proportion of patients needing inpatient hemodialysis transition.

Our study is notable for its large size and the inclusion of veterans from the entire US. To the best of our knowledge, this is the first study to provide a comprehensive characterization of inpatient vs. outpatient hemodialysis transition in a large national cohort of incident hemodialysis patients in the USA. Our study also has several limitations which need to be considered. This being an observational study, we can only report associations, but not cause-effect relationships. We cannot rule out the effects of residual confounding on the observed associations, such as the indication for dialysis initiation or for the hospital admission. Our outcome of interest was all-cause mortality, which does not allow us to examine the reasons why patients died. Our study population consisted of mostly male US veterans, so the result should be applied with caution to females, to non-veterans, or to patients outside the USA.

\section{Conclusions}

A high proportion of incident ESRD patients transition to hemodialysis in an inpatient setting. The reasons behind this may be complex, and likely include both patient-related factors (such as comorbidities), and also potentially modifiable factors, such as lack of pre-transition nephrology care and vascular access creation. Transitioning to hemodialysis in an inpatient setting is also associated with higher mortality, which is in large part explained by a higher comorbidity burden and less ideal preparation for hemodialysis. Transitioning more patients to hemodialysis in an outpatient setting (e.g., by

Inpatient vs. Outpatient Hemodialysis Transition providing more timely pre-transition nephrology care or creation of AVF) could provide cost savings and may also result in better outcomes after transition. The latter hypothesis will need to be tested in prospective clinical trials.

\section{Acknowledgments}

This study is supported by grant 5U01DK102163 from the National Institute of Health to K.K.-Z. and C.P.K., and by resources from the US Department of Veterans Affairs. The data reported have been supplied by the USRDS. Support for VA/CMS data is provided by the Department of Veterans Affairs, Veterans Health Administration, Office of Research and Development, Health Services Research and Development, VA Information Resource Center (Project Numbers SDR 02-237 and 98-004). C.P.K. and K.K.-Z. are employees of the US Department of Veterans Affairs. The interpretation and reporting of these data are the responsibility of the authors and in no way should be seen as official policy or interpretation of the US Department of Veterans Affairs or the US government.

\section{Author Contributions}

Study concept and design: all authors. Acquisition of data: C.P.K., P.K.P., and K.K.-Z. Analysis and interpretation of data: F.M.A., K.S., M.Z.M., P.K.P., J.L.L., F.T., K.K.-Z., and C.P.K. Drafting of the manuscript and approval of the final version: F.M.A. and C.PK. Critical revision of the manuscript for important intellectual content and approval of the final version: all authors.

Each author contributed important intellectual content during manuscript drafting or revision and accepts accountability for the overall work by ensuring that questions pertaining to the accuracy or integrity of any portion of the work are appropriately investigated and resolved. C.P.K. takes responsibility that this study has been reported honestly, accurately, and transparently and that no important aspects of the study have been omitted and that any discrepancies from the study as planned (and, if relevant, registered) have been explained.

Funders of this study had no role in study design; collection, analysis, and interpretation of data; writing the report; and the decision to submit the report for publication.

\section{Statement of Ethics}

The study was approved by the Institutional Review Boards of the Memphis and Long Beach VA Medical Centers, with exemption from informed consent.

\section{Disclosure Statement}

None of the authors have relevant conflicts of interest. 


\section{References}

1 Saran R, Li Y, Robinson B, Abbott KC, Agodoa LY, Ayanian J, Bragg-Gresham J, Balkrishnan R, Chen JL, Cope E, Eggers PW, Gillen D, Gipson D, Hailpern SM, Hall YN, He K, Herman W, Heung M, Hirth RA, Hutton D, Jacobsen SJ, Kalantar-Zadeh K, Kovesdy CP, Lu Y, Molnar MZ, Morgenstern H, Nallamothu B, Nguyen DV, O'Hare AM, Plattner B, Pisoni R, Port FK, Rao P, Rhee CM, Sakhuja A, Schaubel DE, Selewski DT, Shahinian V, Sim JJ, Song P, Streja E, Kurella Tamura M, Tentori F, White S, Woodside K, Hirth RA: US renal data system 2015 annual data report: epidemiology of kidney disease in the United States. Am J Kidney Dis 2016;67(3 suppl 1):Svii, S1-S305.

2 Soucie JM, McClellan WM: Early death in dialysis patients: risk factors and impact on incidence and mortality rates. J Am Soc Nephrol 1996;7:2169-2175.

3 Wong SP, Vig EK, Taylor JS, Burrows NR, Liu CF, Williams DE, Hebert PL, O'Hare AM: Timing of initiation of maintenance dialysis: a qualitative analysis of the electronic medical records of a national cohort of patients from the department of veterans affairs. JAMA Intern Med 2016;176:228-235.

4 Kausz AT, Obrador GT, Arora P, Ruthazer R, Levey AS, Pereira BJ: Late initiation of dialysis among women and ethnic minorities in the United States. J Am Soc Nephrol 2000;11: 2351-2357.

5 Korevaar JC, van Manen JG, Boeschoten EW, Dekker FW, Krediet RT; NECOSAD Study Group: When to start dialysis treatment: where do we stand? Perit Dial Int 2005; 25(suppl 3):S69-S72.

6 Wilson B, Harwood L, Locking-Cusolito H, Chen SJ, Heidenheim P, Craik D, Clark WF: Optimal timing of initiation of chronic hemodialysis? Hemodial Int 2007;11:263269.
7 Rosansky SJ, Cancarini G, Clark WF, Eggers P, Germaine M, Glassock R, Goldfarb DS, Harris D, Hwang SJ, Imperial EB, Johansen KL, Kalantar-Zadeh K, Moist LM, Rayner B, Steiner R, Zuo L: Dialysis initiation: what's the rush? Semin Dial 2013;26:650-657.

8 Cooper BA, Branley P, Bulfone L, Collins JF, Craig JC, Dempster J, Fraenkel MB, Harris A, Harris DC, Johnson DW, Kesselhut J, Luxton G, Pilmore A, Pollock CA, Tiller DJ; IDEAL Study Steering Committee: The Initiating Dialysis Early and Late (IDEAL) study: study rationale and design. Perit Dial Int 2004;24: 176-181.

9 Chen YM, Wang YC, Hwang SJ, Lin SH, Wu KD: Patterns of dialysis initiation affect outcomes of incident hemodialysis patients. Nephron 2016;132:33-42.

10 Watnick S: Financial and medico-legal implications of late-start dialysis: understanding the present policies through a window of past performance. Semin Dial 2013;26:702-705.

11 Molnar MZ, Gosmanova EO, Sumida K, Potukuchi PK, Lu JL, Jing J, Ravel VA, Soohoo M, Rhee CM, Streja E, Kalantar-Zadeh K, Kovesdy CP: Predialysis cardiovascular disease medication adherence and mortality after transition to dialysis. Am J Kidney Dis 2016;68:609-618.

12 Sumida K, Molnar MZ, Potukuchi PK, Thomas F, Lu JL, Jing J, Ravel VA, Soohoo M, Rhee CM, Streja E, Kalantar-Zadeh K, Kovesdy CP: Association of slopes of estimated glomerular filtration rate with post-end-stage renal disease mortality in patients with advanced chronic kidney disease transitioning to dialysis. Mayo Clin Proc 2016;91:196-207.

13 Sumida K, Molnar MZ, Potukuchi PK, Thomas F, Lu JL, Ravel VA, Soohoo M, Rhee CM, Streja E, Yamagata K, Kalantar-Zadeh K, Kovesdy CP: Association between vascular access creation and deceleration of estimated glomerular filtration rate decline in late-stage chronic kidney disease patients transitioning to end-stage renal disease. Nephrol Dial Transplant 2016;pii:gfw220.
14 Kovesdy CP, Norris KC, Boulware LE, Lu JL, Ma JZ, Streja E, Molnar MZ, Kalantar-Zadeh $\mathrm{K}$ : Association of race with mortality and cardiovascular events in a large cohort of US veterans. Circulation 2015;132:1538-1548.

15 Deyo RA, Cherkin DC, Ciol MA: Adapting a clinical comorbidity index for use with ICD-9-CM administrative databases. J Clin Epidemiol 1992;45:613-619.

16 Smith MW, Joseph GJ: Pharmacy data in the VA health care system. Med Care Res Rev 2003;60(3 suppl):92S-123S.

17 Sohn MW, Arnold N, Maynard C, Hynes DM: Accuracy and completeness of mortality data in the Department of Veterans Affairs. Popul Health Metr 2006;4:2.

18 Crews DC, Jaar BG, Plantinga LC, Kassem HS, Fink NE, Powe NR: Inpatient hemodialysis initiation: reasons, risk factors and outcomes. Nephron Clin Pract 2010;114:c19-c28.

19 Fischer MJ, Stroupe KT, Kaufman JS, O’Hare AM, Browning MM, Sohn MW, Huo Z, Hynes DM: Predialysis nephrology care and dialysis-related health outcomes among older adults initiating dialysis. BMC Nephrol 2016; 17:103.

20 McGill RL, Ruthazer R, Meyer KB, Miskulin DC, Weiner DE: Peripherally Inserted Central Catheters and Hemodialysis Outcomes. Clin J Am Soc Nephrol 2016;11:1434-1440.

21 Pisoni RL, Arrington CJ, Albert JM, Ethier J, Kimata N, Krishnan M, Rayner HC, Saito A, Sands JJ, Saran R, Gillespie B, Wolfe RA, Port FK: Facility hemodialysis vascular access use and mortality in countries participating in DOPPS: an instrumental variable analysis. Am J Kidney Dis 2009;53:475-491.

22 Xue H, Ix JH, Wang W, Brunelli SM, Lazarus M, Hakim R, Lacson E Jr: Hemodialysis access usage patterns in the incident dialysis year and associated catheter-related complications. Am J Kidney Dis 2013;61:123-130. 